\title{
Evaluation of a Hot Print System for the development of latent fingermarks on thermal paper
}

Kiera Robb

Paul Deacon

Laura Fordyce

Rebecca Fennessy

Kevin J. Farrugia

This is the accepted manuscript (C) 2019, Elsevier Licensed under the Creative Commons AttributionNonCommercial-NoDerivatives 4.0 International: http://creativecommons.org/licenses/by-nc-nd/4.0/ (c) EY-NC-ND

The published article is available from doi:

https://doi.org/10.1016/j.scijus.2019.08.003 
NOTICE: this is the author's version of a work that was accepted for publication in Science \& Justice. Changes resulting from the publishing process, such as peer review, editing, corrections, structural formatting, and other quality control mechanisms may not be reflected in this document. Changes may have been made to this work since it was submitted for publication. A definitive version was subsequently published in Science \& Justice, DOI: 10.1016/j.scijus.2019.08.003 


\title{
Evaluation of a Hot Print System for the development of latent fingermarks on thermal paper: A pseudo- operational trial.
}

\author{
Kiera Robb ${ }^{1}$, Paul Deacon ${ }^{3}$, Laura Fordyce ${ }^{1}$, Rebecca Fennessy ${ }^{1}$, Kevin Farrugia ${ }^{1,2, *}$ \\ ${ }^{1}$ School of Science, Engineering \& Technology, Division of Science, Abertay University, \\ Bell Street, Dundee, DD1 1HG, UK \\ ${ }^{2}$ Faculty of Health and Life Sciences, Leicester School of Pharmacy, De Montfort \\ University, The Gateway, Leicester, LE1 9BH, UK. \\ ${ }^{3}$ School of Science, Engineering \& Technology, Division of Science, Abertay University, \\ Bell Street, Dundee, DD1 1HG, UK
}

\section{Conflict of interest}

The authors have no conflict of interest to declare.

\section{* Corresponding Author:}

Faculty of Health and Life Sciences,

Leicester School of Pharmacy,

De Montfort University,

The Gateway,

Leicester,

LE1 9BH

kevin.farrugia@dmu.ac.uk 


\section{Highlights}

- The Hot Print System (HPS) is inferior to chemical methods on this substrate.

- The HPS uniquely developed very few number of fingermarks.

- Ninhydrin was the most effective process; however, it compromises text legibility.

- The HPS did not adversely affect subsequent chemical development of fingermarks.

- ThermaNIN formulation modified to include HFE-71DE to avoid precipitation. 


\begin{abstract}
Enhancement of latent fingermarks on thermal paper poses a number of problems when using traditional methods used for porous substrates due to blackening of the thermal layer as a result of polar solvents present within the reagents and high temperatures oxidising the acid/dye complex. Thus, methods which prevent such reactions are favoured for the development of latent prints on said substrates. A comparative pseudo-operational trial using UV, Hot Print System (HPS), ninhydrin and ThermaNIN was performed on 1000 thermal paper substrates gathered from various sources. The results indicated that the most effective method was an acetone pre-wash followed by ninhydrin. The sequence of HPS-ninhydrin was similarly effective when compared to ninhydrin as a sole technique. ThermaNIN produced fewer marks than ninhydrin but was superior to HPS. Whilst the HPS developed some fingermarks, there was only a very small number of marks uniquely developed by it.
\end{abstract}




\section{Introduction}

Thermal paper is a substrate which is used frequently in everyday transactions such as till receipts, tickets, ATM printouts as well as on labels and packaging and can therefore be of great evidential value in criminal investigations and enquiries [1]. Traditionally porous substrates, such as paper, are subjected to chemical enhancement methods such as amino acid sensitive reagents, including ninhydrin and DFO [2]. However, these methods are not suitable for developing fingermarks on thermal paper since blackening of the active layer occurs due to the presence of polar solvents, which oxidise the active coat chemicals hindering visualisation of the original text and any marks developed [3]. The Hot Print System (HPS) by Consolite Forensics claims that the device is a "revolutionary new patented system to automatically and consistently develop fingerprints on thermal paper in minutes, without the use of chemicals". The main advantages of this system is that the text on the thermal paper remains intact and the paper does not turn black.

Thermal papers have a basic design of a substrate, base coat and the active coat which encompasses the fluoran-leuco dye and octadecylphosphonic acid, which oxidises in the presences of heat to form a coloured compound, thus enabling information to be printed onto the paper [4]. The paper may also contain a top coat, which increases the durability of the paper from mechanical wear and protects against environmental and chemical exposure and/or a base coat which protects the paper during printing, prevents heat from penetrating the paper layer as well as protection if an adhesive is to be used [5]. Furthermore, papers may have a double sided active coating, enabling text to be printed thermally on each side [4].

Attempts to solve the problem of the blackening of thermal paper have been previously proposed. Some methods involve the use of direct contact between thermal paper and paper impregnated with chemicals such as 4-dimethylaminocinnamaldehyde (DMAC) and ninhydrin [6-8]. Armitage and Wakefield [3] proposed a solvent free method using low thermal heat applied to the active layer of the paper. They observed $\sim 30^{\circ} \mathrm{C}$ difference in fingermark development temperature on thermal paper to the thermal response temperature, which resulted in the activation of the dye/acid complex and consequently the colour change which is used for printing. They reported that a commercially available, low temperature, thin nozzle hair dryer was the most efficient at developing fingermarks on the thermal paper, without background interference; however, the developed fingermarks faded rapidly depending on the brand of paper. Schwarz et al [9] introduced a two-step process which reversed blackening of the receipts using $\mathrm{G} 3$ solution; however, the whitening affect deteriorated over time and the two-step procedure was time consuming. A further method proposed the use of 
polyvinylpyrrolidones (PVP) to be incorporated with ninhydrin solution in an attempt to stabilise the whitening affect in a one-step processes [10].

Bond [1] developed a low temperature thermal application instrument which formed a uniform distribution of heat resulting in the development of fingermarks on the thermal side of investigated papers. It was reported that the developed marks did not undergo fading as observed in another study [3] and further proposed that the type of paper would influence the development. In addition, it was proposed that polar amino acids such as lysine, increased the solubility of the leuco-dye and reduced the temperature in which the coloured complex forms. A later study by Bond [11] compared the application of chemical treatment ThermaNIN to the thermal application using the Hot Print System by Consolite ${ }^{\circledR}$ Forensics Ltd., as well as the effect of heat treatment on sequential processes using ninhydrin. The study reported that HPS is not donor specific and that the enhancement exploiting the leuco dye is less sensitive to reduced sweat deposits than traditional chemical methods. It was concluded that the application of heat to thermal papers results in more ridge detail than chemical applications and it did not hinder subsequent treatment of ninhydrin for the development of marks on the reverse side of the papers. They proposed that heat treatment was a faster more efficient method for the development of fingermarks on these substrates than the twelve hours required for ThermaNIN treated papers. It is important to note that this was a study involving a number of donors using planted split marks. Another study by Bond [12] reports that the use of UV light (peak 365nm) is a suitable way for a speculative, non-destructive way of detecting fingermarks on thermal paper. As the properties of thermal papers appear to vary between different manufacturers and countries, the use of UV was reported as more efficient in the detection of latent fingermarks on thermal paper.

Treatment of thermal paper with normal formulations of the amino acid reagent 1,2-indanedione (1,2IND) will generally result in darkening of the paper and limit the observation of latent fingermarks. The Australian Federal Police and other studies have reported other formulations as well as drycontact treatment with 1,2-IND [6]. Goel [13] compared indanedione-zinc (IND-Zn) dry contact method to HPS where it was reported that HPS was inferior to the IND-Zn dry contact method, which developed high quality continues ridge detail fingermarks over the time periods investigated. It was also proposed that IND-Zn was also more cost efficient despite the increased development time required. Another study [14] compared 1,2-Indanedione (1,2-IND) and ThermaNIN, for both "idealistic"-planted fingermarks and "realistic" fingermarks which included tickets, receipts and labels. They concluded that both techniques were capable of developing fingermarks on the substrates tested. Fingerprint examiners preferred 1,2-IND over ThermaNIN in terms of clarity; however, also noted that an alternative light source would be required to view the fluorescent 1,2-IND marks. A comparison of ninhydrin, DFO and 1,2-IND for the detection of latent marks on train tickets (consisting 
of a thermal printed layer and a cellulose paper side) reported the superiority of 1,2-IND over the other two techniques [15]. Another study reported that ninhydrin was superior to ThermaNIN in the detection of latent marks on thermal paper [16].

Presently, the recommended development technique for the development of fingermarks on thermal papers in the United Kingdom, according to the Fingermark Visualisation Manual [17], is a visual examination followed by capture of the text if it is evidentially important. This is followed by fluorescence examination followed by an acetone pre-wash (which removes the thermal layer) prior to the sequential treatment of DFO, ninhydrin and physical developer. CAST noted that ninhydrin may result in less marks than DFO or 1,2-IND; however, the process enables quick and efficient evaluation of developed marks without the requirement for an alternative light source. According to the Fingermark Visualisation Manual [17], ThermaNIN is a category C process which is described as a method at the development stage and exhibits potential as an effective technique for the recovery of fingermarks. This process should be applied when retention of the printed information is vital. Nonetheless, this technique may be detrimental to subsequent fingermark recovery methods as well as other forensic processes. ThermaNIN is a hemiketal and converts to ninhydrin and alcohol in the presence of water either in the atmosphere or within the paper substrate, the alcohol and ninhydrin formed react with amino acids within the fingermark residues.

The research undertaken in this study was a pseudo operational trial of 1000 thermal paper receipts. CAST [18] defines these trials as "to establish whether the results obtained in laboratory trials are replicated on articles/ surfaces typical of those that may be submitted to a fingerprint laboratory, or to distinguish between closely equivalent formulations that cannot be separated in laboratory trials". The pseudo-operational trial of a substantial amount of 1000 items further ensures that results observed during laboratory trials are also matched under pseudo and later full-operational settings. The methods investigated in this study included: optical examination under natural and UV-A light; thermal application using the Hot Print System (HPS) and chemical treatment using ninhydrin and ThermaNIN. Some sequential processes were also investigated. The techniques were primarily used to determine which of the techniques were most efficient at developing and enhancing fingermarks on the thermal side of the samples, methods which also developed fingermarks on the reverse (paper) side of the receipts were also recorded. The main aim of the study was to evaluate if the HPS technique can develop unique latent fingermarks not developed by other techniques used in this study. The base technique of ninhydrin was selected for comparison purposes rather than the best available technique. It is almost certain that 1,2-indanedione will find more marks than ninhydrin and the use of physical developer at the end of the sequence, although inferior to amino acid techniques, will find additional new marks. 


\section{Method and Materials}

The date of issue and retailer of each receipt was recorded followed by sectioning receipts into thirds for all the three processes undertaken in this study. Each section was numbered and allocated a letter categorising the process to be undertaken. A was assigned to the Hot Print System (HPS), B signified ninhydrin and $\mathbf{C}$ represented ThermaNIN (figure 1). The designated processes were rotated to ensure that each technique was used for each area of receipt, thus minimising any bias. Visual and fluorescent examination was carried out prior to any treatment. A grading scheme for pseudo-operational trials was used as recommended by CAST [18] and the International Fingerprint Research Group [19] where any ridge detail greater than $64 \mathrm{~mm}^{2}$ was included as a detection. The first 500 thermal receipts were treated as described in Figure 1 and the next 500 receipts had a slight change whereby the sequence in Process A was changed to HPS-ThermaNIN rather than HPS-ninhydrin.

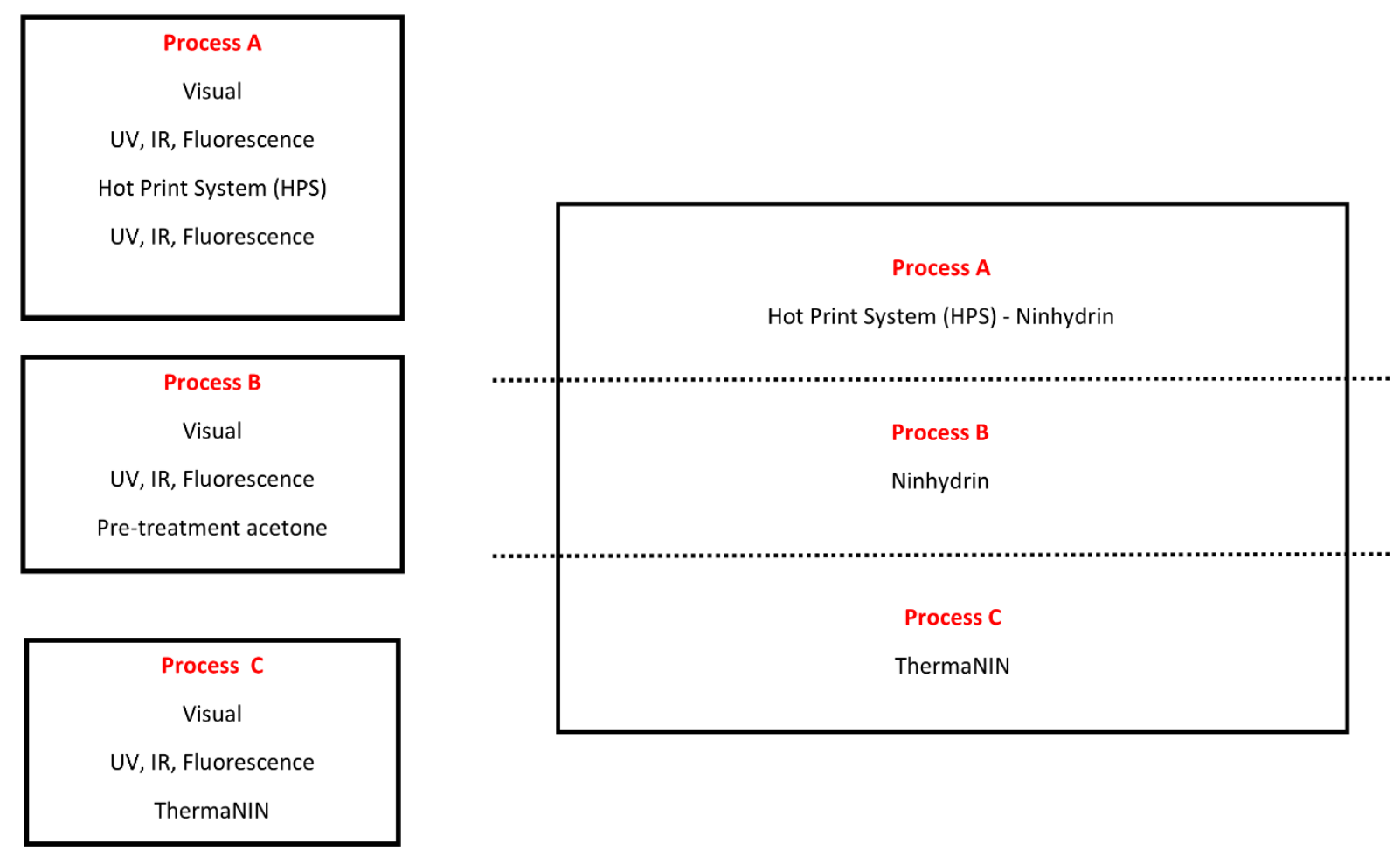

Figure 1 - Sample division for a substrate in the study.

\section{Fluorescence Examination}

All thermal paper samples were viewed under fluorescence of varying wavelengths using a Quaser 2000 and Crime-Lites ${ }^{\circ} 825$ with the recommended viewing filters. Such an example was exciting with a blue Crime-Lite $82 \mathrm{~S}$ ( $10 \%$ band width $420-470 \mathrm{~nm}$ with a $445 \mathrm{~nm}$ peak) and viewing with a yellow long pass $476 \mathrm{~nm}$ filter ( $1 \%$ cut-on point). Furthermore, a Labino UV light (peak $325 \mathrm{~nm}$ and viewed with a clear UV filter) was also used. An Integrated Rapid Imaging System (Home Office, IRIS) was used 
for IR examination with the associated tungsten lamp and consideration of various long-pass filters: $630,645,695,715,780,850$ and $1000 \mathrm{~nm}$. Marks observed were circled and reviewed after each process. Photography was performed with a Nikon D5100 and a 60mm micro Nikon lens.

\section{Hot Print System}

The Hot Print System (HPS) was operated as recommended by the manufacturer. The system was switched on ensuring the power button LED was green, each receipt was processed individually for the system to detect contrast on the surfaces of the thermal layer. The heatwave symbol would flash orange during the contrast detection phase after pushing the start button. A completion signal emitted from the system indicated the end of the contrast detection and the receipt removed. The HPS then proceed with the cooling cycle, indicated by a solid orange light on the heatwave symbol on the front of the machine. Once the cooling system was complete the heatwave symbol would turn green, which indicated that the next receipt could be processed.

\section{Ninhydrin}

A concentrated solution of ninhydrin was prepared by dissolving ninhydrin ( $5 \mathrm{~g}$, Sigma Aldrich) in ethanol ( $45 \mathrm{~mL}$, Fisher), acetic acid ( $5 \mathrm{~mL}$, Fisher) and ethyl acetate ( $2 \mathrm{~mL}$, Fisher). A working solution was then prepared by adding HFE-7100 (1 L, 3M Novec) to ninhydrin concentrated solution ( $52 \mathrm{~mL})$.

Using forceps, each receipt was placed into a metallic dish containing acetone in order to remove the thermal active layer and air dried within the fume cupboard. Some receipts required additional washes in order to completely remove the active layer. The dish was cleaned regularly in between samples and fresh acetone added. Using a corrugated dipping trough, the receipts were then passed through the ninhydrin working solution and air-dried. The receipts were then placed into a humidity oven at $80^{\circ} \mathrm{C}$ and $65 \% \mathrm{RH}$ for 4 minutes. The receipts were then observed after processing and within $24 \mathrm{hrs}$ of development with frequent examination over the next 2 weeks. The receipts were stored in the dark at ambient temperature throughout the study.

\section{ThermaNIN}

The manufacturer (BVDA) recommends a working solution prepared by dissolving $0.4-0.5 \mathrm{~g}$ of ThermaNIN and dissolved in propan-2-ol (0.5 mL, Fisher) and ethyl acetate (1.5 mL, Fisher) before adding HFE7100 (100 mL, 3M Novec). This resulted in precipitation of the ThermaNIN and the carrier solvent was changed to HFE-7100 (100 mL, 3M Novec) and HFE-71DE (25 mL, 3M Novec). Working solutions were prepared weekly and stored in dark bottles. The receipts were then placed onto cardboard trays and allowed to dry. These were then placed into a cabinet with a humidity of about 
$80 \%$ and left in the dark overnight. The receipts were then examined within 72 hours and stored in dark conditions.

HPS Sequential Processes (Process A)

After treatment with the HPS, the developed prints were examined and any deterioration was noted.

The first batch (Sequence 1) of 500 receipts was treated in sequence with Ninhydrin (UV-HPS-NIN). The second batch (Sequence 2) of 500 receipts was treated in sequence with ThermaNIN (UV-HPSThermaNIN).

Evaluation of the effects of repeated HPS cycles

Fifty thermal paper receipts were subjected up to five repeated HPS cycles and any observations were noted.

Evaluation of the effects of ageing

Fifty thermal paper receipts aged between 3-6 years were included in the trial to observe the effects of ageing on the observed enhancement. 


\section{Results \& Discussion}

Fluorescence (visible light spectrum) and IR examination, before and after treatments, did not visualise any latent fingermarks.

\section{Sequence 1}

Figure 2 represents the number of fingermarks developed by each process as well as the sequential process of HPS treated receipts with NIN. Initial observations with UV revealed 57 marks; 9 of which were also enhanced with HPS and 51 with ninhydrin. Three marks were uniquely developed by UV visualisation. Treatment with ninhydrin required an acetone wash which removed all the printed information and provided 232 marks on the thermal side but also 208 marks on the rear side. Ninhydrin also developed 17 out of the 19 HPS prints indicating that two marks were uniquely developed by the HPS. For process B, ninhydrin developed 239 fingermarks on the thermal side and also 78 marks on the reverse (cellulose) side. Ninhydrin developed most of the marks observed under UV. For process $C$, the ThermaNIN kept the printed information intact and developed 146 fingermarks on the thermal side, and 98 fingermarks on the reverse side. Furthermore, 44 out of the 55 UV observed marks were also enhanced with ThermaNIN.

Fingermarks which had been developed using the HPS process and then placed in an acetone pretreatment were eradicated due to the removal of the thermal coating; however, if ninhydrin did enhance these marks, the Ruhemann's purple development would be present within the pencil-circled area. In some cases ninhydrin, as well as, ThermaNIN treatment would further enhance the marks out with the circled area potentially providing additional ridge detail. Although, the number of fingermarks detected by HPS was low, sequential treatment with ninhydrin detected a considerable amount of new marks. Nonetheless, when ninhydrin was used on its own (process B) more marks were detected than the sequential process A (UV-HPS-NIN). 


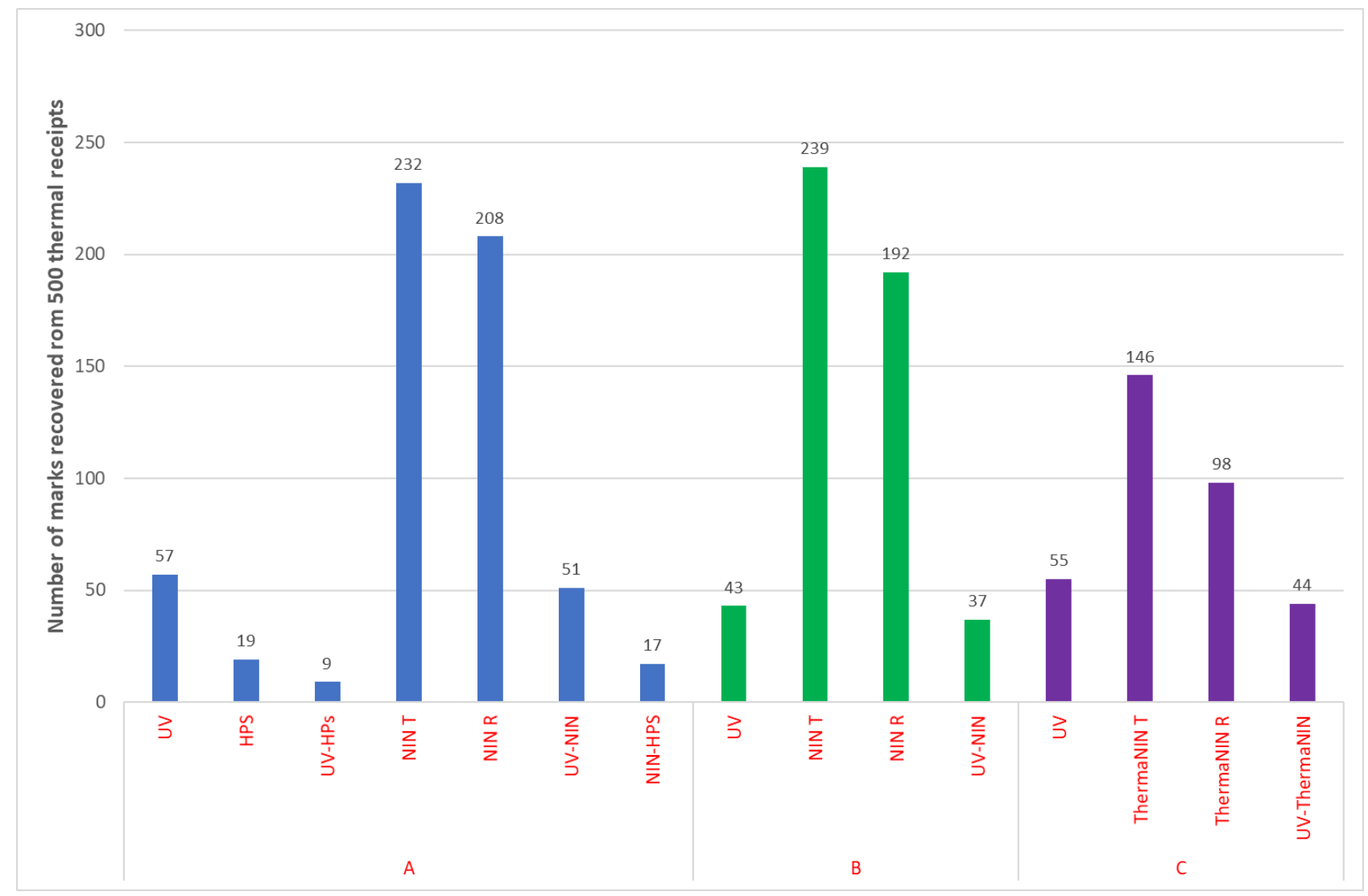

Figure 2 - Total number of fingermarks developed by each process in Sequence 1

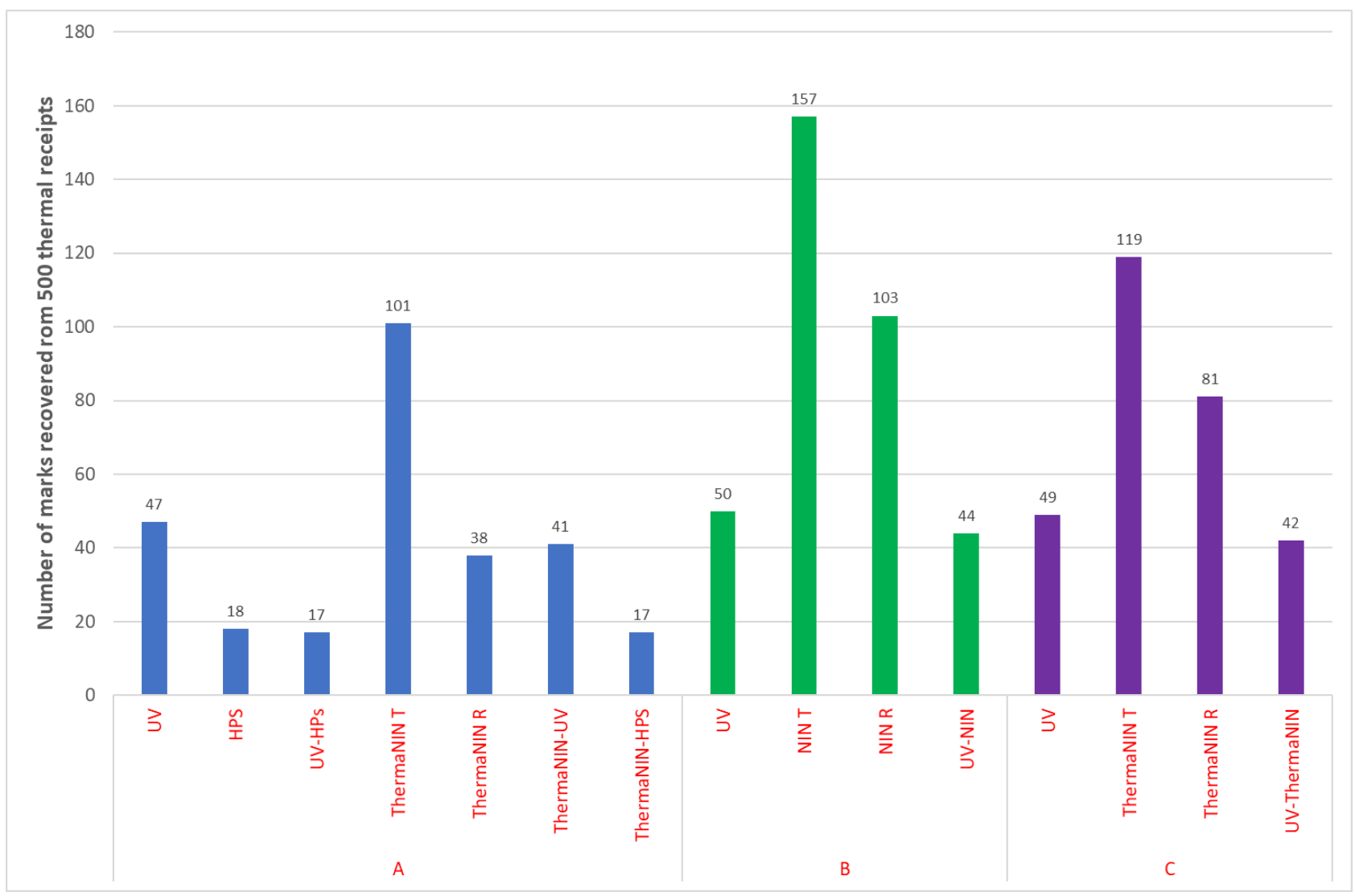

Figure 3 - Total number of fingermarks developed by each process in Sequence 2 


\section{Sequence 2}

The second sequence was processed as sequence 1; however, for process $A$ the sequential process was performed with ThermaNIN rather than with ninhydrin. Figure 3 represents the total number of fingermarks enhanced by each process.

In process A, HPS enhanced 17 out of the 47 marks observed under UV which were in turn also developed with ThermaNIN. Further development of HPS treated marks using ThermaNIN resulted in an additional 101 marks on the thermal side and 38 on the reverse side. Most of the UV observed marks were also developed with ThermaNIN; however, three marks were only found with the use of UV. Similarly to sequence 1 , there was only one mark that were unique to the HPS process. In process B, ninhydrin enhanced 44 out of the 50 UV marks, with a total of 157 marks observed on the thermal side and a further 103 marks on the reverse side. In process C, ThermaNIN enhanced 42 out of the 49 UV observed marks, and a total of 119 fingermarks on the thermal side and 81 on the reverse side.

\section{Comparison of techniques}

Overall, UV-A light was an effective initial non-destructive technique for the visualisation of latent fingermark on thermal paper. Around $10 \%$ of the marks were uniquely developed by UV visualisation highlighting its value in the sequential enhancement of fingermarks. A further advantage of the nondestructive UV-A examination was that when a parking ticket, where some of the text had faded, some of the text could be visualised. Figure 4 shows an example of a latent fingermark developed with UVA on a bus ticket. A recent report [20] highlighted the visualisation of latent fingermarks on Italian highway tickets by illuminating with UV-A $(\lambda=365 \mathrm{~nm})$ and collection of the emitted radiation with a long-pass filter fixed at $693 \mathrm{~nm}$. This method did not reveal any fingermarks in this study, possibly due to the difference in the thermal paper quality between highway tickets and store receipts.

HPS was outperformed by the other processes investigated with a very small number of marks uniquely found by the technique. The fingermarks developed were often weak to visualise and deteriorated over time. Figure 5 represents an example of HPS developed fingermarks on thermal paper. A major drawback for HPS is that only one receipt can be processed at any time within the system and the time for processing each receipt varied, with some taking over 2 minutes, in addition to a subsequent $\sim 2$-minute cooling cycle, until the next receipt could be inserted onto the detection platform. Thus, the process can become time consuming, where large volumes of papers to be analysed is required. A further consideration is that both sides of the receipts may be handled, HPS 
can only detect marks on the thermal side of the paper and therefore subsequent processes would need to be considered to maximise the recovery of fingermarks on this type of substrate.

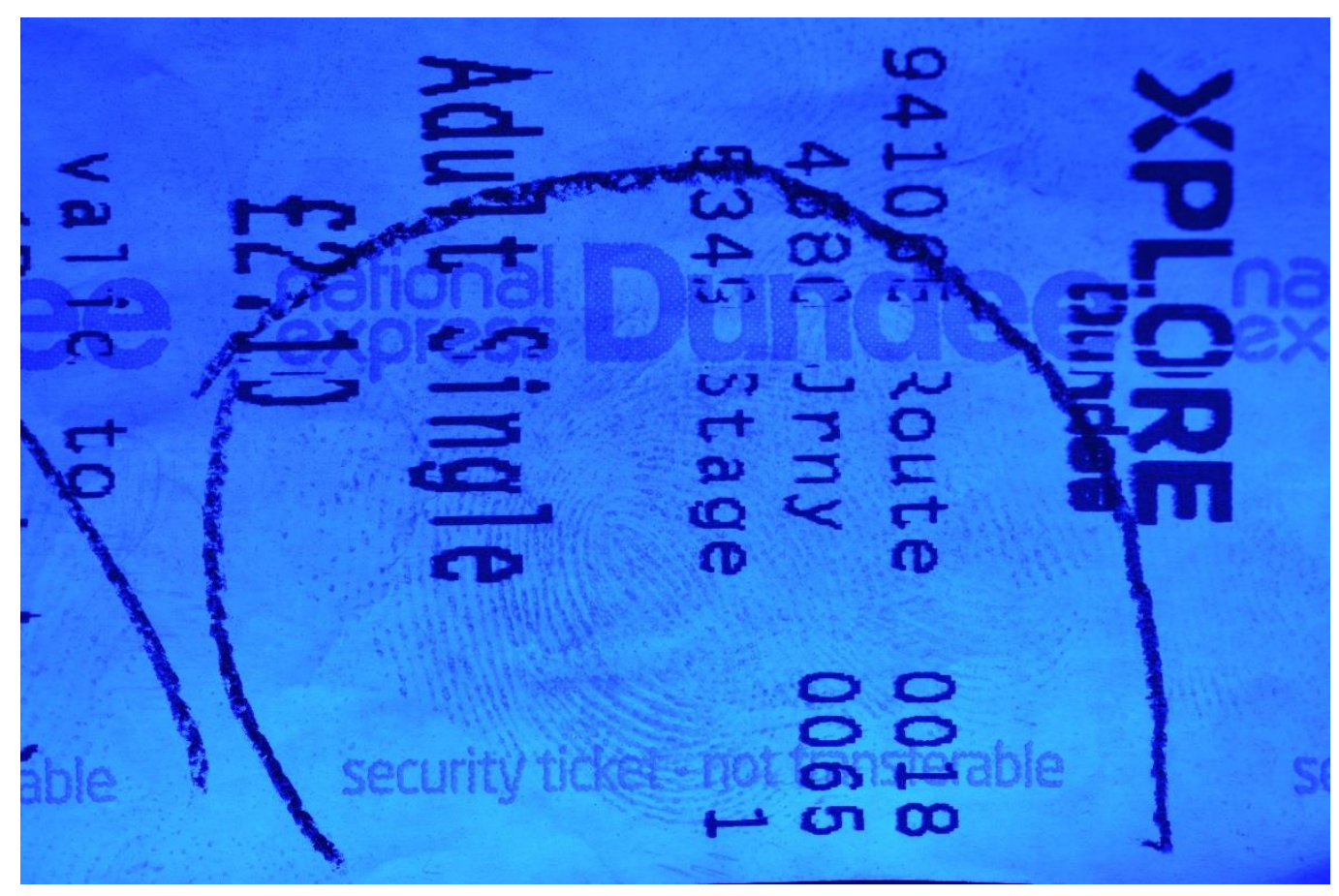

Figure 4 - Latent fingermark developed with UV-A and viewed with a UV filter

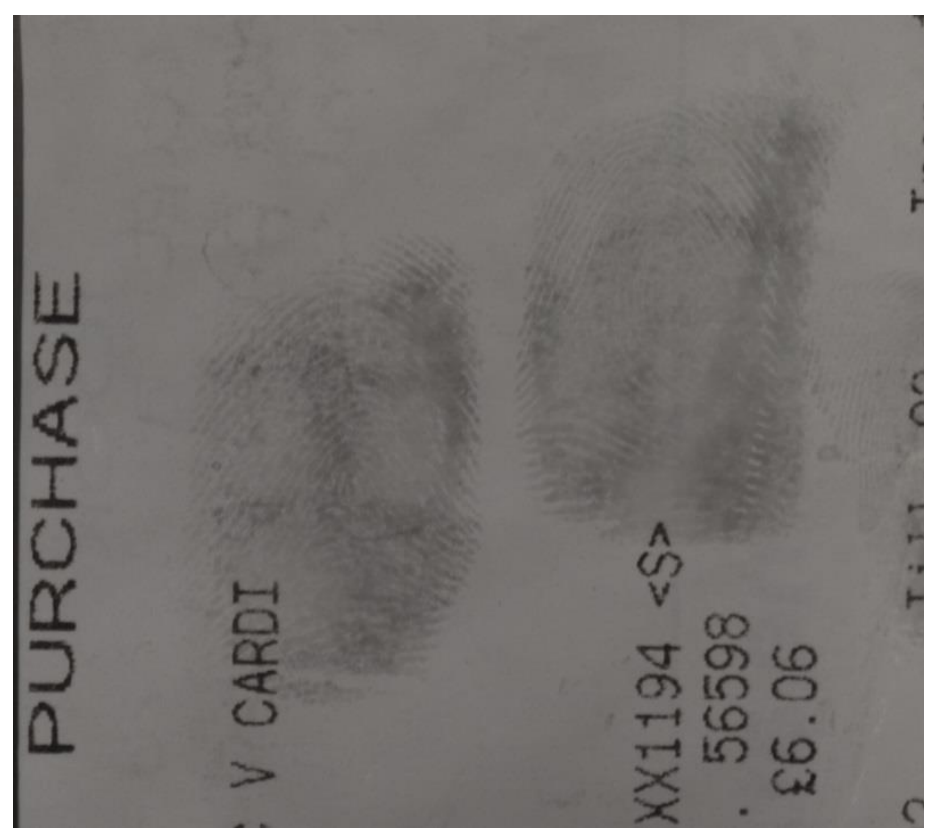

Figure 5 - Latent fingermark developed with HPS under white light 
ThermaNIN enhanced fingermarks on both the thermal side and reverse side of the receipts donated, in addition to the preservation of the printed text (figure 6). The results produced on the thermal side of the paper had a light pink-purple colouration and at times background development occurred, which hindered contrast between the developed marks. The reverse (cellulose) side resulted in a purple/blue colour, which at times also resulted in weak development. The ThermaNIN formulation recommended by the manufacturer BVDA using HFE7100, isopropanol and ethyl acetate resulted in ThermaNIN precipitating out of solution. Therefore, an amended solution comprising of isopropanol, ethyl acetate, HFE7100 (75\%) and HFE71DE (25\%) was used to which no precipitation nor discolouration of the receipts occurred. When using the revised formulation, if a receipt was to be passed through the dipping tray of solution twice blackening of the receipts occurred therefore the receipts were only passed through the solution once, with care taken to ensure wetted receipts did not come into contact with those drying. BVDA does suggest additional treatment with ThermaNIN; however, advised that the items are allowed to dry fully prior to a second process. Furthermore, the commercial BVDA products INON and ThermaNIN can occasionally be wrongly discussed in the literature as the same product. The attached alcohol in ThermaNIN is decanol whereas for INON it is 3,5,5-trimethyl-hexanol (isonanol).

Ninhydrin resulted in the greatest number of marks developed both individually and sequentially. Examples of ninhydrin developed marks are presented in figure 7. Although ninhydrin had the greater ability to develop fingermarks on both the thermal and reverse (cellulose) side of the samples, the processes is destructive due to the obliteration of the text on the thermal side. On re-examination of some of the marks using UV-A light source, some text was legible on the thermal side despite the removal of visual text by the acetone prewash. Other studies have indicated that the text may be visualised post text removal [2,21].

A key observation is that the sequence HPS-ninhydrin provided a similar number of detections as when ninhydrin was solely used. Another study also reported that the HPS did not inhibit subsequent development with ninhydrin [11]. The sequence HPS-ThermaNIN provided a lower detection rate than when ThermaNIN was solely used. 

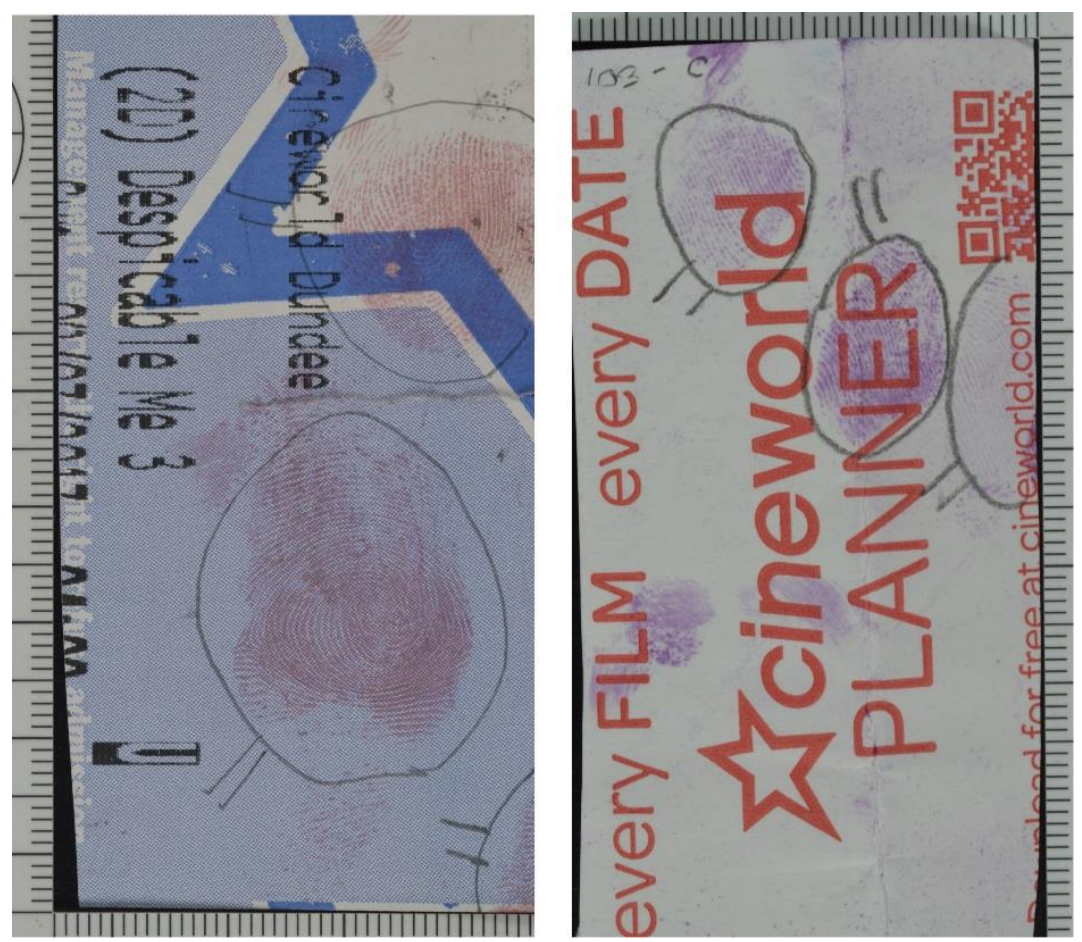

Figure 6 - ThermaNIN developed fingermarks on thermal side (left) and reverse side (right)
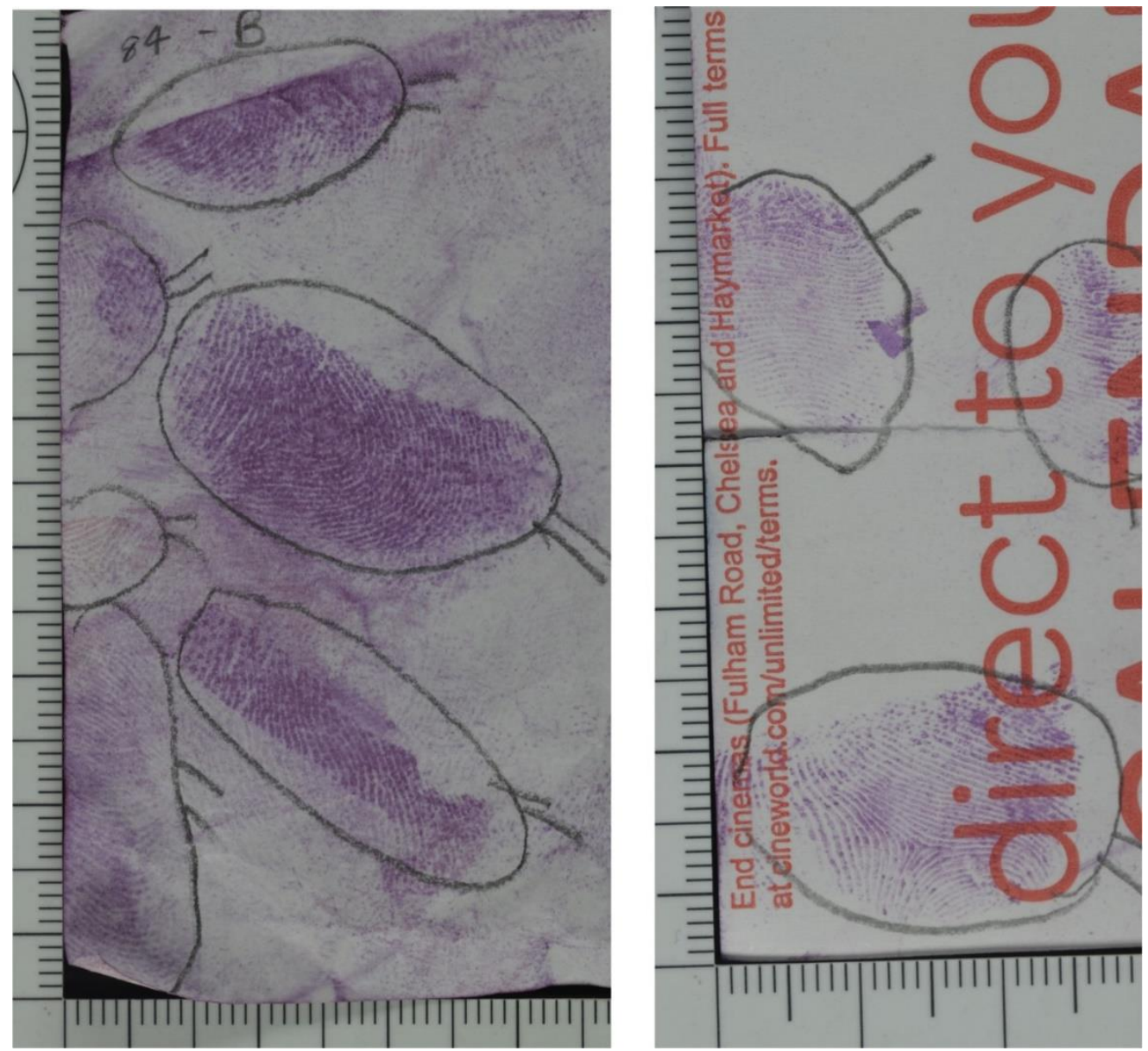

Figure 7 - Ninhydrin developed fingermarks on thermal side (left) and reverse side (right). 
Evaluation of the effects of repeated HPS cycles and the effects of ageing

Consolite ${ }^{\circledR}$ Forensics Ltd. suggest that receipts can undergo several HPS cycles, and encourage this if no marks are developed after the first cycle. In this study, no additional detail was observed following additional cycles in line with a previous study [13]. There was no effects of blackening after additional HPS cycles. The trends observed for sequences 1 and 2 were also observed on the aged thermal receipts.

\section{HPS device problems}

Of the marks detected with HPS, the ridges appeared black with slight discolouration to the background. For some of the receipts the processing cycle had to be stopped prior to completion due to an error occurring, in which two audio signals were emitted from the instrument and the power LED light changed from green to red, whilst the heatwave light continued to flash orange. Initially when this occurred the receipts were removed and the "contrast detection mode" was allowed to go to completion as dictated by the device and the cooling cycle had completed, before the receipt was replaced into the HPS and a secondary cycle was initiated. If frequent occurrences of the said error occurred, a test sample, using the thermal paper provided by Consolite ${ }^{\circledR}$ Forensics Ltd., was placed into the system to check operation. Each time the HPS would detect the planted marks, and consequently the rest of the receipts would be processed, as well as the receipt in which the error occurred. On subsequent processing of some of the receipts to which the error occurred, background development sometimes resulted which posed a problem for visualising any fingermarks. Blackening also occurred on some receipts where an error during processing did not occur. The user manual advised in the troubleshooting section that if the LED light was orange/red the device should be switched off and on again. The literature did not specify if this procedure should be followed if the error occurred during the contrast detection phase or whether the advice was for the initial start-up. Some receipts were double sided, thus if fingermarks were present, they were expected to be developed on both sides, yet when the second side was processed with HPS, blackening of the entirety of the receipt resulted. The resultant blackening of the receipt during the second processing, consequently resulted in a diminished contrast, thus visualisation between any developed fingermarks and the background was hindered. 


\section{Conclusion}

The pseudo-operational trial of 1000 thermal receipts aimed to evaluate the ability of the Hot Print System developed by Consolite ${ }^{\circledR}$ Forensics Ltd. for the development of latent fingermarks on thermal paper and to compare the technique to other chemical enhancement methods recommended by CAST for the development of fingermarks on thermal paper. The techniques included ninhydrin, ThermaNIN as well as sequential process using HPS followed by ninhydrin and ThermaNIN.

The results from this study suggest that the HPS is inferior to UV, ninhydrin and ThermaNIN. Whilst the HPS developed some fingermarks, there was only a very small number of marks uniquely developed by it. Furthermore, the process can be lengthy as thermal papers have to be individually examined. As 1,2-indanedione will soon be a category A process in the Fingermark Visualisation Manual, future work will involve further comparisons using different formulations and delivery methods. The use of physical developer at the end of an enhancement sequence will find additional new marks and a new physical developer formulation using decaethylene glycol mono- dodecyl ether (DGME) will also be assessed. 


\section{References}

[1] J.W. Bond, Development of Latent Fingerprints on Thermal Paper by the Controlled Application of Heat, J. Forensic Sci. 58 (2013) 767-771.

[2] L. Schwarz, I. Klenke, Enhancement of ninhydrin- or DFO-treated latent fingerprints on thermal paper, J. Forensic Sci. 52 (2007) 649-655.

[3] M. Wakefield, S. Armitage, Development of latent fingerprints on thermal paper using a novel, solvent free method, J. Forensic Identif. 55 (2005) 202-213.

[4] R.S. Ramotowski, ed., Lee and Gaensslen's Advances in Fingerprint Technology, 3rd ed., CRC Press, Florida, 2013.

[5] J.T. Stimac, Thermal \& Carbonless Papers : A Fundamental Understanding for Latent Friction Ridge Development, J. Forensic Identif. 53 (2003) 185-197.

[6] E.L.T. Patton, D.H. Brown, S.W. Lewis, Detection of latent fingermarks on thermal printer paper by dry contact with 1,2-indanedione, Anal. Methods. 2 (2010) 631-637.

[7] H. Marquez, Technique for Processing Carbonless Documents for Latent Prints, J. Forensic Identif. 49 (1999).

[8] J.L. Lee, S. Bleay, V.G. Sears, S. Mehmet, R. Croxton, Evaluation of the Dimethylaminocinnemaldeyhde Contact Transfer Process and its Application to Fingerprint Development on Thermal Papers, J. Forensic Identif. 59 (2009) 545-568.

[9] L. Schwarz, Using Indanedione-Zinc, Heat, and G3 Solution Sequentially to Detect Latent Fingerprints on Thermal Paper, J. Forensic Identif. 61 (2011) 30-37.

[10] L. Schwarz, I. Klenke, Improvement in latent fingerprint detection on thermal paper using a one-step ninhydrin treatment with polyvinylpyrrolidones (PVP)., J. Forensic Sci. 55 (2010) 1076-9.

[11] J.W. Bond, Comparison of Chemical and Heating Methods to Enhance Latent Fingerprint Deposits on Thermal Paper, J. Forensic Sci. 59 (2014) 485-489.

[12] J.W. Bond, A Noninvasive and Speculative Method of Visualizing Latent Fingerprint Deposits on Thermal Paper, J. Forensic Sci. 60 (2015) 1034-1039.

[13] T.L. Goel, Developing Latent Fingermarks on Thermal Paper : Comparison of the 1,2Indanedione-Zinc Chloride Dry Contact Method to the Hot Print System, J. Forensic Identif. 65 
(2015) 34-43.

[14] M. Ponschke, M. Hornickel, A Limited Validation and Comparison of 1, 2-Indanedione and ThermaNin for Latent Print Development on Thermal Paper, J. Forensic Identif. 66 (2016) 245-258.

[15] M. Levin-Elad, Y. Liptz, K.L. Bar-Or, J. Almog, 1,2-Indanedione - A winning ticket for developing fingermarks: A validation study, Forensic Sci. Int. 271 (2017) 8-12.

[16] T. Fitzi, R. Fischer, S. Moret, A. Becue, Fingermark Detection on Thermal Papers : Proposition of an Updated Processing Sequence, J. Forensic Identif. 64 (2014) 329-350.

[17] Centre for Applied Science and Technology (CAST), Fingermark Visualisation Manual, Home Office U.K, 2014.

[18] V.G. Sears, S.M. Bleay, H.L. Bandey, V.J. Bowman, A methodology for finger mark research, Sci. Justice. 52 (2012) 145-160.

[19] IFRG, Guidelines for the Assessment of Fingermark Detection Techniques, J. Forensic Identif. 64 (2014) 174-200.

[20] P. Cappiello, F. Zampa, M. Siciliano, B. Amata, R. Di Palma, M. Modica, A.P. Lazzaro, Visualization of Fingermarks Deposits on Untreated Thermal Paper Exploiting the Near Infrared Luminescence, J. Forensic Sci. (2019) 1556-4029.14071.

[21] P.F. Kelly, R.S.P. King, S.M. Bleay, T.O. Daniel, The recovery of latent text from thermal paper using a simple iodine treatment procedure., Forensic Sci. Int. 217 (2012) e27-30. 